\title{
Seeing right through you: Applications of optical imaging to the study of the human brain
}

\author{
GABRIELE GRATTON, ${ }^{\mathrm{a}}$ MONICA FABIANI, ${ }^{\mathrm{a}}$ THOMAS ELBERT, ${ }^{\mathrm{b}}$ AND \\ BRIGITTE ROCKSTROH ${ }^{\mathrm{b}}$ \\ ${ }^{a}$ Department of Psychology and Beckman Institute, University of Illinois at Urbana-Champaign, Urbana, Illinois, USA \\ ${ }^{\mathrm{b}}$ Department of Behaviorial Neuroscience and Biomagnetism, University of Konstanz, Konstanz, Germany
}

\begin{abstract}
A new set of techniques allows for the study of brain function by near-infrared light, exploiting two optical phenomena: Changes in light absorption are determined by changes in the concentration of substances like oxy- and deoxyhemoglobin, and changes in light scattering occur as a consequence of variations of properties of membranes and corpuscles in the neural tissue. Methods based on light absorption can be used to study hemodynamic changes in the brain, whereas those based on light scattering can be used to study neuronal activity and to provide anatomical information at a cellular and subcellular level. Three optical imaging approaches can be used to study living tissue: reflection, optical coherence tomography (OCT), and photon migration. These three approaches vary in their penetration (from less than a millimeter for reflection to up to $3-5 \mathrm{~cm}$ for photon migration) and spatial resolution (from a micron level for reflection and OCT to a millimeter and centimeter level for photon migration). This issue includes a collection of articles reviewing applications of these technologies to the study of brain and other bodily functions in humans.
\end{abstract}

Descriptors: Optical imaging, Cognitive neuroscience, Brain imaging

This special issue of Psychophysiology includes eight articles (in addition to this overview) aimed at highlighting a set of techniques for studying the human brain based on the use of near-infrared light from a psychophysiological perspective. These techniques have all been developed within the last decade or so and have opened new possibilities for exploring brain function. Optical techniques have several advantages: They can image both neuronal and hemodynamic activity, and, in some cases, anatomical details. They are safe, can be adapted to a number of experimental and practical conditions, and are easy to combine with other techniques [such as electrophysiological, neuromagnetic, and magnetic-resonance (MR) imaging methods]. The major limitation of these techniques is due to the limited penetration of light into living tissue. This precludes measures being taken from structure located at more than $5 \mathrm{~cm}$ distance from the surface of the head (or from the location from which measures are taken). Assume that only one in a billion photons (light particles) penetrates from the light source through tissue to a detector and assume further that the source emits $10^{20}$

This work was supported by grants from NIMH (MH57125; G. Gratton, PI), DARPA (NSF EIA 00-79800; G. Gratton \& M. Fabiani, PIs), NIA (AG21887-01; M. Fabiani, PI), and the Optik Zentrum, University of Konstanz to Thomas Elbert and Brigitte Rockstroh.

Address reprint requests to: Gabriele Gratton, University of Illinois, Beckman Institute, 405 N. Matthews Ave., Urbana, IL 61801 USA. Email: grattong@uiuc.edu. photons - a brief exposure to sunlight, for instance: Then the signal will still be a billion photons. Given that modern devices can detect single photons, it is obvious that the signal may provide rich sources of information along different dimensions such as photon density, traveling time, phase, dispersion of wave packages, interference, and so forth. How valuable these different types of information actually are for imaging bodily and brain functions remains an exciting experimental adventure for the years to come.

The idea that changes in the way living tissue absorbs, reflects, or scatters light may provide information about psychological states or processes is very old (e.g., Darwin, 1872). We can all easily recognize signs of emotional states (sudden pallor or blushing) or of exertion (redness and perspiration) in other people just observing their faces-perhaps the simplest way to make optical observations. What is novel about the set of techniques reviewed in this special section is that they allow us to measure changes of a relatively similar nature occurring not only in the skin but in deeper bodily structures such as the brain.

This "window into the brain" is made possible by the use of near-infrared light, which penetrates several centimeters into the living tissue. This occurs because the major absorbing substances in the human body (water, oxy- and deoxy-hemoglobin, and melanin) absorb relatively little light at wavelengths between 690 and 1,000 nm (near-infrared "window"). If light in this range is used, the main factor limiting photons crossing thick tissue (such 
as the human head) is that the tissue is not only absorbing but also highly scattering. Scattering properties vary quite substantially between different types of tissue: At one extreme are blood and cerebrospinal fluid (which scatter light very little), and at the other extreme is white matter (which is very highly scattering; see Taddeucci, Martelli, Barilli, Ferrari, \& Zaccanti, 1996). The scattering properties of other important head tissues such as skin, bone, and gray matter are in between these two extremes (Taddeucci et al.). However, even these substances are sufficiently scattering to make it difficult for near-infrared light to penetrate more than a few centimeters into the head. Still, a vast proportion of cortical areas can be reached by near-infrared photons illuminating the surface of the head, thus providing an effective avenue for noninvasive optical measurements of human cortical function.

\section{Optical Signals}

Optical imaging methods allow us to visualize the interaction of photons with tissue. There are four major types of such interactions: (a) absorption (i.e., the energy of the photon is transferred to the tissue, typically in the form of heat), (b) phosphorescence (i.e., the energy of the photon is released by the tissue with a delay in the form of a photon with similar-or lower-energy), (c) fluorescence (i.e., the energy of the photon is released in part in the form of a lower energy photon, the rest being dissipated as heat or as another lower energy photon), and (d) scattering (i.e., the photon remains of the same energy and is merely deviated in its trajectory). Of course, another alternative is that the photon proceeds undisturbed in its trajectory (which means that the tissue is transparent for a particular photon); however, this cannot really be considered as a photon-medium interaction.

All these types of interactions may (and do) occur to some degree in the brain and in other living tissue; however, for nearinfrared light, the most commonly occurring are scattering and absorption. Further, for both scattering- and absorption-type interactions, the frequency of occurrence may change as a function of neuronal activity. Changes in light scattering in relationship to action potentials are well documented (Cohen, 1972; Stepnoski et al., 1991). They are typically attributed to changes in the neuronal membrane reflectance due to the reorientations of molecules in the membrane and/or to volumetric changes that are determined by the movement of ions and water across and around the membrane. Changes in scattering and absorption properties are also likely to occur in the dendrites and soma during postsynaptic potentials. At the macroscopic level, the postsynaptic potentials, reflecting the activity of a much more extended portion of the neurons and integrating the activity over longer periods of time, are likely to dominate the measures. Several studies indicate that neural tissue becomes more transparent (i.e., less scattering) when it is activated and probably less transparent (i.e., more scattering) when it is inhibited, in a manner that appears very similar in time course to the local field potential recorded from the same area (Frostig, Lieke, Ts'o, \& Grinvald, 1990; Rector, Harper, \& George, 2002; Rector, Poe, Kristensen, \& Harper, 1997).

Changes in light absorption are instead related more directly to the changes in the blood oxygenation level that occur within a few seconds after the activation of a particular cortical area. Both oxy- and deoxy-hemoglobin have very characteristic and distinct absorption spectra, which, in fact, correspond to the different coloration of arterial and venous blood. This makes it possible to use a spectroscopic approach to measure changes in the concentration of oxy- and deoxy-hemoglobin as a function of neural activity (Grinvald, Lieke, Frostig, Gilbert, \& Wiesel, 1986; Malonek \& Grinvald, 1996). Several phenomena have been thus demonstrated, such as a small increase in deoxyhemoglobin concentration occurring within $1 \mathrm{~s}$ from the onset of neural activity (reflecting the increased oxygen consumption of active neural tissue), which is soon followed by a decrement in deoxy-hemoglobin concentration and a much larger increase in the oxy-hemoglobin concentration (corresponding to the BOLD fMRI signal and dependent on vasodilation; Malonek \& Grinvald).

The absorption changes are much larger than the scattering changes: Absorption changes related to vasodilation may result in changes of the signal amplitude of the order of $1-2 \%$, whereas the scattering effects related to neuronal events may change the signal by about $0.01-0.1 \%$ [compare, e.g., the effects reported by Franceschini, Fantini, Thompson, Culver, and Boas (this issue) and by Maclin, Gratton, and Fabiani (this issue)]. Thus, the hemodynamic-related absorption changes are much easier to observe than the neuronal phenomena. However, the scattering changes are a direct indication of neural activity, and therefore possess inherently better temporal (and perhaps spatial) resolution than the hemodynamic phenomena. In addition, different types of measurement methods may bias the recording system more toward one or the other of the two signals. In this issue, three of the articles deal with the small but fast scattering signals, and four of the articles are related to measurement of the much larger but slower absorption signals.

\section{Approaches for Optical Brain Imaging}

There are three possible approaches that allow for the measurement of optical properties in vivo (see Figure 1). First, we can illuminate an extended portion of the tissue and study the light that is reflected back (or back-scattered) to some recording instrument (such as a charge-coupled device camera or an array of light-sensitive diodes) that is pointed toward the illuminated surface. As demonstrated by animal research (see Frostig, 2002, for a review) and observations during surgery (see Toga \& Pouratian, 2002), this relatively simple measurement apparatus can be used to provide very useful information, in particular regarding changes in blood flow to various cortical areas. Under appropriate conditions, the measurement can have very high spatial and even notable temporal resolution. When this measurement approach is used, a large proportion of the reflection comes from the very first interaction of the photons with the illuminated tissue, which, because of the scattering characteristics of the tissue itself, will occur within 1-2 $\mathrm{mm}$ of the surface. This first "reflection" will produce highly coherent images that can, in fact, be used to construct very detailed maps. A proportion of the photons will, as mentioned above, penetrate further into the tissue. However, because these photons will "bounce" around the tissue quite a bit before reemerging at the surface, they will tend to create an incoherent image, which will fade in the background of the coherent image generated by the initial reflection, and will be difficult to visualize. Also, the probability that photons following such a longer path will 


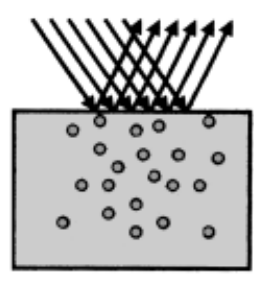

Reflection

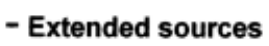

- Extended detectors

- High spatial resolution

- Limited penetration

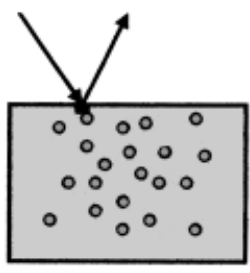

OCT

- Small sources

- Small detectors

- Cancellation/interference - Less spatial resolution

- High spatial resolution - Deeper penetration

- Reduced penetration

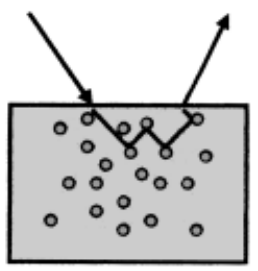

Photon Migration

- Small sources

- Small detectors

- Fully non-invasive

Figure 1. Schematic representation of the penetration of the three most commonly used approaches to optical imaging: reflection, optical coherence tomography (OCT), and photon migration. Other characteristics of the three techniques are also described.

actually reemerge in the direction of the photo-detector decreases rapidly with the length of the photon path, both because of the stochastic nature of the scattering process and because of an increased probability of absorption (because the probability of absorption is related to the length of the path followed by the photons in the medium). As a consequence, images of scattering media obtained using reflection methods are almost entirely measures of surface phenomena. This means that, for all practical purposes, cortical measurements obtained with reflection methods are limited to cases in which the cortex is exposed (in animals or in humans during surgery) or to cases in which the measurements are taken from very close locations (as when the skull is surgically thinned or in newborn infants). Therefore, these measures, although very useful in a number of cases, do not really fall into the realm of psychophysiology, which, by definition refers to measurements that are taken noninvasively. We have therefore opted not to present this type of measurement in this special issue and instead refer the interested reader to excellent reviews of this area, such as that provided by Frostig (2002) in a recent book.

A second method for examining the optical properties of tissue in vivo is optical coherence tomography (OCT). This approach is reviewed in this issue in a article by Steve Boppart, an investigator who has made important contributions to this area. OCT is based on principles of interference and cancellation of near-infrared waves penetrating into tissue. As for the reflection methods, it is based on the first interaction (back-scattering) of photons in tissue. Unlike the reflection measures, however, it directly visualizes the depth at which this first interaction occurs. In combination with scanning methods, it can, therefore, provide three-dimensional information about the scattering properties of living tissue. The spatial resolution that can be reached is excellent (micron level), which makes OCT suitable for in vivo biopsy, allowing for the visualization of cell membranes and nuclei even without a contrast agent or dye. At present, the temporal resolution is limited mostly by the time required to acquire an image. This process can be very fast (millisecond level) if the field of view is limited, but may require hundreds of milliseconds or seconds for extended fields of view. Technical advances can be expected to greatly improve this aspect of the technique. Because OCT is based on imaging the back-scattering from tissue, it can be expected to be sensitive to fast, neuronal signals related to scattering, although a direct investigation of this property of OCT has not yet been attempted. A major drawback of OCT for psychophysiological studies is its limited penetration (at most a few millimeters). Because signals from different depths are separated by the measurement approach, OCT can detect deeper events more easily than a reflection approach. The dependence on the first interaction of photons with the medium imposes maximum penetration limits on the technology that are incompatible with using this approach to study the human brain in a noninvasive manner. However, OCT could, in principle, be used to study the human brain invasively during surgical operations or through implanted devices and catheters, and, in a completely noninvasive fashion, to study the functioning of the retina.

The vast majority of the articles contained in this special issue are based on a third approach to optical imaging: photon migration. Photon migration allows investigators to image relatively deep structures in highly scattering media. The logic of this approach is to use an array of small light sources and detectors. The light emitted by the sources diffuses in a random fashion through the scattering medium. However, because of statistical properties, the photons picked up by the detectors are more likely to have followed certain paths than others through the medium. If enough photons are used, the entire process of migration of photons from the sources to the detectors can be described quite accurately using mathematical formulations related to the diffusion process. This makes it possible to go beyond images of the first interaction of photons with the medium and therefore to produce images of structures that are up to a few centimeters deep into the medium (at present, the deepest functional data correspond to depth of $2.5 \mathrm{~cm}$ from the surface of the head; Gratton, Sarno, Maclin, Corballis, \& Fabiani, 2000). Of course, the images, by virtue of their being related to a stochastic diffusion process, possess much less spatial resolution than those obtained with reflection or OCT. However, the spatial resolution can be improved by using multiple source-detector combinations exploring the same area (provided that the source emitting the light reaching any particular detector can be identified; see, e.g., Gratton \& Fabiani, Maclin et al., and Wolf et al., all in this issue) and can vary depending on the specific measurement method used. One of the articles presented in this issue (Gratton \& Fabiani) estimates the spatial resolution 
(i.e., minimum distance with negligible cross talk) of photon migration methods using this multiple source-detector method to be approximately $1.5 \mathrm{~cm}$. This makes photon migration methods comparable to PET and fMRI in terms of spatial resolution. This level of spatial resolution is sufficient to identify major cortical subdivisions (such as different Brodmann areas) and in some cases gross functional maps of particular areas. For instance, Gratton and Fabiani show how optical imaging data can be used to separate responses from medial occipital areas related to different quadrants of the visual field (see also Gratton, Corballis, Cho, Fabiani, \& Hood, 1995; Gratton et al., 1997, 2000, for similar types of measures).

\section{The Articles in This Issue}

Of the eight articles of this special issue, one (Boppart) presents an overview of OCT, and seven present empirical and review articles related to photon migration methods. Of these, three articles (Gratton \& Fabiani; Maclin et al.; Wolf et al.) are investigations focusing on the "fast" scattering signal, whereas the other four articles (Franceschini et al.; Hebden; Hoshi; Matsuo et al.) focus on "slow," hemodynamic signals.

The emphasis on photon migration methods reflects the applicability of this technology to noninvasive studies of the human brain. With respect to other technologies for studying brain function, photon migration methods have several advantages and some limitations, several of which are reviewed in the various manuscripts. In summary, photon migration methods have very low invasivity, because they are based on small amounts on nonionizing radiation. They do not require subjects to be immobilized, as the equipment is relatively light, and optic fibers can be used as conduit to and from the head. Because an optical (rather than an electrical or magnetic) technology is used, it is very easy to eliminate environmental sources of interference, and the measures are compatible with other technologies (see, e.g., Toronov et al., 2001, for examples of simultaneous recording of photon-migration and MR data, and Gratton et al., 1997; Gratton, Goodman-Wood, \& Fabiani, 2001; and Rinne et al., 1999, for simultaneous recording of photon-migration and EEG/ ERP data). The combination of these properties means that optical methods can be applied to most subject populations and recording environments. This makes these measures particularly useful with children (as shown in the review presented by Hebden) and clinical and applied settings (as in the article by Matsuo et al.). They provide a centimeter-level spatial resolution (as shown by Franceschini et al. and Gratton \& Fabiani). The measures can be obtained practically continuously, allowing for the study of the time course of neuronal (as in Gratton \& Fabiani; Maclin et al.; and Wolf et al.) and/or hemodynamic (Franceschini et al.; Hebden; Hoshi; Matsuo et al.) events. The possibility of measuring simultaneously both neuronal and vascular signals is in fact one of the major advantages of optical imaging, because of the possibility of bridging across the two major families of imaging methods currently used (electrophysiological and hemodynamic methods). Work in this area has, in fact, already been carried out in several studies (see above). Another advantage of photon migration is related to the possibility of measuring separately the concentration of oxyand deoxy-hemoglobin (see Franceschini et al. and Hoshi), which in some cases are functionally dissociated. Hoshi reviews the use of different techniques for recording photon migration data, contrasting in particular continuous-wave methods with time-resolved methods. The latter methods appear superior in terms of accuracy of quantification properties, although, of course, at the cost of making the response system more complex and expensive.

The major limitation of photon migration methods is their penetration ( $3-5 \mathrm{~cm}$ at most) that makes it impossible to study structures located deep into the brain. Compounded with the low transparency of white matter (Taddeucci et al., 1996), this limitation makes current noninvasive optical imaging a technology that is appropriate for cortical but not subcortical structures.

Another important current limitation of optical imaging, in particular for the fast, scattering signal, is the relatively low signal-to-noise ratio. Two articles in this issue (Maclin et al. and Wolf et al.) describe methodologies that can be used to improve the signal-to-noise ratio of fast signals. The Maclin et al. study reports the use of frequency filters, whereas the Wolf et al. article focuses on recording (pi-detectors) and analysis (cross-correlation) methods. In both cases, marked improvements in signal-tonoise ratio are achieved. In addition, other approaches for improving signal-to-noise ratio, based on redesigning the recording instrument to avoid time-multiplexing and increasing source intensity, are currently under development. It is therefore possible to imagine that measurements with a much better signalto-noise ratio could be available in a few years. Indeed, improvements in signal-to-noise ratio may also make it possible for measurements to be extended to deeper structures than is presently feasible.

Compared to other imaging techniques, optical imaging, when performed on a routine basis, will be inexpensive and safe. It can be added easily in standard psychophysiological laboratories and even be included in ambulatory measurements. Given the rapid developments in quantum optics and optical techniques, such as the possibility to produce light pulses in the femtoseconds $\left(10^{-15} \mathrm{~s}\right)$ range or to employ multiple dynamic light scattering, completely new ways of optical imaging appear on the horizon and together with the already existing approaches promise a bright future for psychophysiology.

\section{REFERENCES}

Boppart, S. A. (2003). Optical coherence tomography: Technology and applications for neuroimaging. Psychophysiology, 40, 529-541.

Cohen, L. B. (1972). Changes in neuron structure during action potential propagation and synaptic transmission. Physiological Review, 53, 373-417.

Darwin, C. (1872). The expression of the emotions in man and animals. London: John Murray.

Franceschini, M. A., Fantini, S., Thompson, J. H., Culver, J. P., \& Boas, D. A. (2003). Hemodynamic evoked response of the sensorimotor cortex measured noninvasively with near-infrared optical imaging. Psychophysiology, 40, 548-560.

Frostig, R. D., Lieke, E. E., Ts'o, D. Y., \& Grinvald, A. (1990). Cortical functional architecture and local coupling between neuronal activity and the microcirculation revealed by in vivo high-resolution optical imaging of intrinsic signals. Proceedings of the National Academy of Sciences, USA, 87, 6082-6086.

R. D. Frostig (Ed.). (2002). In vivo optical imaging of brain function. Boca Raton, FL: CRC Press. 
Gratton, G., \& Fabiani, M. (2003). The event-related optical signal (EROS) in visual cortex: Replicability, consistency, localization, and resolution. Psychophysiology, 40, 561-571.

Gratton, G., Corballis, P. M., Cho, E., Fabiani, M., \& Hood, D. (1995). Shades of gray matter: Noninvasive optical images of human brain responses during visual stimulation. Psychophysiology, 32, 505-509.

Gratton, G., Fabiani, M., Corballis, P. M., Hood, D. C., GoodmanWood, M. R., Hirsch, J., Kim, K., Friedman, D., \& Gratton, E. (1997). Fast and localized event-related optical signals (EROS) in the human occipital cortex: Comparison with the visual evoked potentials and functional MRI. NeuroImage, 6, 168-180.

Gratton, G., Sarno, A. J., Maclin, E., Corballis, P. M., \& Fabiani, M. (2000). Toward non-invasive 3-D imaging of the time course of cortical activity: Investigation of the depth of the event-related optical signal (EROS). NeuroImage, 11, 491-504.

Gratton, G., Goodman-Wood, M. R., \& Fabiani, M. (2001). Comparison of neuronal and hemodynamic measure of the brain response to visual stimulation: An optical imaging study. Human Brain Mapping, 13, 13-25.

Grinvald, A., Lieke, E., Frostig, R. D., Gilbert, C. D., \& Wiesel, T. N. (1986). Functional architecture of cortex revealed by optical imaging of intrinsic signals. Nature, 324, 361-364.

Hebden, J. C. (2003). Advances in optical imaging of the newborn infant brain. Psychophysiology, 40, 501-510.

Hoshi, Y. (2003). Functional near-infrared optical imaging: Utility and limitations in human brain mapping. Psychophysiology, 40, 511-520.

Maclin, E. L., Gratton, G., \& Fabiani, M. (2003). Optimum filtering for EROS measurements. Psychophysiology, 40, 542-547.

Malonek, D., \& Grinvald, A. (1996). Interactions between electrical activity and cortical microcirculation revealed by imaging spectroscopy: Implications for functional brain mapping. Science, 272, 551-554.

Matsuo, K., Kato, T., Taneichi, K., Matsumoto, A., Ohtani, T., Hamamoto, T., Yamasue, H., Sakano, Y., Sasaki, T., Sadamatsu, M., Iwanami, A., Asukai, N., \& Kato, N. (2003). Activation of the prefrontal cortex to trauma-related stimuli measured by near-infrared spectroscopy in posttraumatic stress disorder due to terrorism. Psychophysiology, 40, 492-500.

Rector, D. M., Harper, R. M., \& George, J. S. (2002). In vivo observations of rapid scattered-light changes associated with electrical events. In R. D. Frostig (Ed.), In vivo optical imaging of brain function (pp. 93-112). Boca Raton, FL: CRC Press.

Rector, D. M., Poe, G. R., Kristensen, M. P., \& Harper, R. M. (1997). Light scattering changes follow evoked potentials from hippocampal Schaeffer collateral stimulation. Journal of Neurophysiology, 78, $1707-1713$.

Rinne, T., Gratton, G., Fabiani, M., Cowan, N., Maclin, E., Stinard, A., Sinkkonen, J., Alho, K., \& Näätänen, R. (1999). Scalp-recorded optical signals make sound processing from the auditory cortex visible. NeuroImage, 10, 620-624.

Stepnoski, R. A., LaPorta, A., Raccuia-Behling, F., Blonder, G. E., Slusher, R. E., \& Kleinfeld, D. (1991). Noninvasive detection of changes in membrane potential in cultured neurons by light scattering. Proceedings of the National Academy of Sciences, USA, $88,9382-9386$.

Taddeucci, A., Martelli, F., Barilli, M., Ferrari, M., \& Zaccanti, G. (1996). Optical properties of brain tissue. Biomedical Optics, 1, $117-123$.

Toga, A. W., \& Pouratian, N. (2002). Intraoperative optical imaging. In R. D. Frostig (Ed.), In vivo optical imaging of brain function (pp. 173-192). Boca Raton, FL: CRC Press.

Toronov, V., Webb, A., Choi, J. H., Wolf, M., Michalos, A., \& Gratton, E. (2001). Investigation of human brain hemodynamics by simultaneous near-infrared spectroscopy and functional magnetic resonance imaging. Medical Physics, 28, 521-527.

Wolf, M., Wolf, U., Choi, J. H., Toronov, V., Paunescu, L. A., Michalos, A., \& Gratton, E. (2003). Fast cerebral functional signal in the $100-\mathrm{ms}$ range detected in the visual cortex by frequency-domain near-infrared spectrophotometry. Psychophysiology, 40, 521-528.

(RECEIVED October 20, 2002; ACCEPTED December 10, 2002) 